\title{
MINERÍA DE FLUJOS DE DATOS: UN MAPEO SISTEMÁTICO DE FUENTES SECUNDARIAS
}

\author{
María Yesenia Zavaleta-Sánchez y Edgard Benítez-Guerrero \\ Universidad Veracruzana, Xalapa, Ver., México
}

\begin{abstract}
RESUMEN
Un flujo de datos (data stream en Inglés) es una secuencia de datos, posiblemente infinita, que se genera continuamente en el tiempo para ser almacenada o procesada por un sistema. Ejemplos de estos son los que se generan en transacciones bancarias, redes sociales, sensores, correo electrónico, entre otros. El procesamiento y análisis de flujos de datos es cada vez más demandado para obtener información y generar conocimiento en tiempo real sobre algún tema de interés. El objetivo principal de este trabajo es identificar las técnicas de minería de flujos de datos más utilizadas en Journals de tipo Survey y las áreas de oportunidad para el desarrollo de trabajos futuros. Para esto se realizó un mapeo sistemático de documentos recuperados de bases de datos científicas. Como resultado se obtuvo que la detección de patrones frecuentes, agrupación y clasificación son las técnicas de minería de flujos de datos más discutidas.
\end{abstract}

\section{PALABRAS CLAVE}

Flujo de Datos, Minería de Flujos de Datos, Mapeo Sistemático

\section{INTRODUCCIÓN}

Un flujo de datos (data stream en Inglés) es una secuencia de datos que se genera de forma continua, posiblemente de forma infinita, para ser procesada (Abdelguerfi, et al., 2005; Koudas y Srivastava, 2005). Ejemplos de flujos de datos son los que provienen de sensores, sistemas de vigilancia, transacciones bancarias, navegación en la web, redes sociales, sistemas meteorológicos, monitores de signos vitales en hospitales, procesos de marketing, mercados de valores, entre otros (Ellis, et al., 1991). En las aplicaciones de tipo stream los datos se generan en tiempo real; además, la tasa de llegada y el volumen de los datos es tan grande que es impráctico su almacenamiento, por lo que su tratamiento se ejecuta normalmente sobre la marcha. Por ende, la forma de captarlos, procesarlos y analizarlos debe ser distinta a las formas convencionales. Para analizar flujos de datos, comúnmente se utilizan técnicas de minería de datos (data mining en Inglés). Estas buscan identificar conocimiento, en forma de patrones de comportamiento de los datos, que sirva de ayuda en la toma de decisiones (Molero y Meda, 2010). Las técnicas tradicionales de minería, no obstante, requieren para trabajar de datos previamente almacenados. Sin embargo, las características inherentes a los flujos de datos en términos de tiempo y memoria hacen que las técnicas de análisis de datos tradicionales no pueden ser aplicadas de igual forma (Adedoyin-Olowe, et al., 2013; Alothali, et al., 2019). Estas deben adaptarse para considerar la evolución de los datos en el tiempo y trabajar con datos en tan solo una pasada, es decir, luego de que el dato llega y se procesa, éste generalmente caduca y no se tiene disponible para análisis posteriores (Jiang y Gruenwald, 2006; Hulten, et al., 2001). El objetivo de este artículo es identificar tendencias en las temáticas abordadas en la minería de flujos de datos a través de un mapeo sistemático de fuentes de información secundarias (documentos tipo "Survey"). Un mapeo sistemático de la literatura es un método definido para construir clasificaciones y realizar análisis temáticos con el fin de tener un mapa visual del conocimiento existente dentro de un tema amplio (Petersen, et al., 2008). Las categorías utilizadas en un estudio de mapeo generalmente se basan en información de las publicaciones (nombres de autores, afiliaciones de autores, fuente de publicación, tipo de publicación, fecha de publicación, etc.) y/o información sobre los métodos de investigación utilizados (Kitchenham, et al., 2011). El resto de este documento está organizado de la siguiente manera. La sección 2 describe el método de investigación, la sección 3 presenta los resultados y las discusiones de la investigación realizada, mientras que la sección 4 presenta las conclusiones del trabajo. 


\section{MÉTODO DE INVESTIGACIÓN}

Los pasos esenciales del proceso de nuestro estudio de mapeo sistemático fueron la definición de preguntas de investigación, la búsqueda sistematizada de trabajos relevantes en bases de datos científicas, la selección de trabajos, análisis del contenido de los artículos y los resultados del mapeo.

\subsection{Preguntas de Investigación}

RQ1. ¿Cuántas publicaciones de tipo Survey en Minería de Flujos de datos se han realizado desde el año 2000? RQ2. ¿Cuáles son las técnicas de la Minería de datos que se utilizan para analizar flujos de datos? RQ3. ¿Cuáles son las áreas pendientes por abordar que dan pauta al desarrollo de trabajos futuros?

\subsection{Proceso de Búsqueda}

Se utilizaron las bases de datos científicas: ACM Digital Library, IEEE Xplore, ScienceDirect y SpringerLink. La cadena de búsqueda utilizada fue: "(SURVEY OR REVIEW OR OVERVIEW OR STATE OF THE ART) AND DATA AND STREAM AND MINING”. Criterios de inclusión: Journals en inglés a partir del 2000 hasta el 2019. Como resultados iniciales se encontraron 926 artículos (113 ACM, 67 IEEE Explore, 92 ScienceDirect y 654 en Springer). Criterios de exclusión: Después de una revisión detallada de títulos, resumen, palabras clave y contenido, se excluyeron publicaciones no relacionadas directamente con el tema, quedando 14 artículos de tipo Survey: 2 ACM, 1 IEEE Explore, 4 ScienceDirect y 7 en Springer (ver Tabla 1).

Tabla 1. Resultados de la búsqueda

\begin{tabular}{cccc}
\hline $\begin{array}{c}\text { Bases de } \\
\text { datos }\end{array}$ & Cadena de búsqueda & $\begin{array}{c}\text { Artículos } \\
\text { encontrados }\end{array}$ & $\begin{array}{c}\text { Artículos } \\
\text { elegidos }\end{array}$ \\
\hline ACM & & 113 & 2 \\
IEEE Explore & “(SURVEY OR REVIEW OR OVERVIEW OR STATE OF & 67 & 1 \\
ScienceDirect & THE ART) AND DATA AND STREAM AND MINING” & 92 & 4 \\
SpringerLink & THE & 654 & 7 \\
\hline Total & & 926 & 14
\end{tabular}

\section{RESULTADOS Y DISCUSIÓN}

En esta sección, de forma general, se responde a las preguntas de investigación planteadas en la sección 2.1 y se discuten brevemente los resultados obtenidos. RQ1. En la Tabla 1 se muestran los resultados del procedimiento de búsqueda. Sólo se seleccionaron 14 artículos de impacto para este trabajo. La publicación más antigua es del 2007 y la más reciente del 2019. RQ2. En la Tabla 2 se observa que la detección de patrones frecuentes (Frequent pattern en Inglés) es la técnica de la minería de datos que se discute con mayor frecuencia en estos artículos y la cual implementa algoritmos incrementales para calcular los patrones frecuentes de las transacciones más recientes (Han, et al., 2007; Cheng, et al., 2008; Manerikar y Palpanas, 2009; Liu, et al., 2011; Gama, 2012; PhridviRaj y GuruRao, 2014; Nasreen, et al., 2014). En segundo lugar, se ubica la agrupación de flujos de datos (Clustering en Inglés) y la cual tiene como objetivo agrupar un conjunto de datos en subconjuntos (grupos), donde los objetos dentro de cada grupo son "similares" y los objetos entre grupos son "diferentes" con respecto a una medida de similitud dada (Gama, 2012; Silva, et al., 2013; Nguyen, et al., 2015; Ghesmoune, et al., 2016; Mohammadi, et al., 2018; Kolajo, et al., 2019). También se han elaborado publicaciones donde se abordan las técnicas de clasificación (Classification en Inglés), cuyo proceso consiste en encontrar un modelo general a partir de datos etiquetados conocidos y luego usar este modelo para predecir etiquetas de clase para nuevos objetos de datos (Gama, 2012; Nguyen, et al., 2015; Gomes, et al., 2017; Krawczyk, et al., 2017; Mohammadi, et al., 2018). 
Tabla 2. Técnicas de Minería de Flujos de Datos

\begin{tabular}{|c|c|c|}
\hline Técnica de Minería & Referencia & Total \\
\hline Agrupación & $\begin{array}{l}\text { (Gama, 2012), (Silva, et al., 2013), (Nguyen, et al., 2015), (Ghesmoune, } \\
\text { et al., 2016), (Mohammadi, et al., 2018), (Kolajo, et al., 2019) }\end{array}$ & 6 \\
\hline Clasificación & $\begin{array}{l}\text { (Gama, 2012), (Nguyen, et al., 2015), (Gomes, et al., 2017), (Krawczyk, } \\
\text { et al., 2017), (Mohammadi, et al., 2018) }\end{array}$ & 5 \\
\hline $\begin{array}{l}\text { Detección de Patrones } \\
\text { Frecuentes }\end{array}$ & $\begin{array}{l}\text { (Han, et al., 2007), (Cheng, et al., 2008), (Manerikar y Palpanas, 2009), } \\
\text { (Liu, et al., 2011), (Gama, 2012), (PhridviRaj y GuruRao, 2014), } \\
\text { (Nasreen, et al., 2014) }\end{array}$ & 7 \\
\hline
\end{tabular}

RQ3. Las características dinámicas de los flujos de datos requieren algoritmos adaptativos a los cambios en las condiciones ambientales y en las necesidades del usuario. Los algoritmos de minería de datos de próxima generación deben preocuparse por la gestión de costo-rendimiento y las limitaciones en todos los aspectos de los recursos computacionales (Gama, 2012). Probablemente el mayor desafío en la agrupación de flujo de datos es construir algoritmos sin introducir parámetros críticos, como el número esperado de grupos o la densidad esperada de los grupos. Otros desafíos son el tratar con estructuras de datos complejas y con valores atípicos, el detectar cambios en la distribución de datos y dar seguimiento a la evolución de clústeres para predecir el clúster al que pertenecerá un dato futuro. Los flujos de texto son más complicados de analizar, ya que no están estructurados, contienen un alto nivel de ruido y existen en diferentes formatos, por lo que la minería de flujos de texto sigue siendo una tarea pendiente por abordar para detectar eventos frecuentes en línea (Nguyen, et al., 2015; Ghesmoune, et al.,2016; Kolajo, et al., 2019).

\section{CONCLUSIONES}

A través del mapeo sistemático de publicaciones de tipo Survey se pudo constatar que la minería de flujos de datos se ha convertido en un área de investigación de mayor interés en los últimos años debido a la importancia de sus aplicaciones y a la creciente generación de datos con diferentes estructuras. Sin embargo, debido a la naturaleza de los flujos de datos, el extraer conocimiento e identificar patrones ocultos en tiempo real, enfrenta muchos desafíos y restricciones. La detección de patrones frecuentes, agrupación y clasificación son las técnicas de minería de datos para las cuales se han discutido más trabajos de tipo Survey. Sin embargo, no son las únicas técnicas de la minería de flujos que se han adaptado de la minería de datos tradicional. Finalmente, cabe destacar que los resultados de este trabajo forman la parte inicial de una investigación más profunda. Aquí sólo se incluyeron jornals de algunas bases de datos científicas debido a la profundidad con la que se aborda la temática de interés. Sin embargo, existen otras fuentes como Google Académico donde se encuentran otro tipo de publicaciones en los cuales, se incluye a las series de tiempo como una técnica de análisis para flujos de datos (Gaber, 2005; Ikonomovska, et al., 2007; Gaber, et al., 2014).

\section{AGRADECIMIENTO}

Este trabajo fue apoyado parcialmente por el Consejo Nacional de Ciencia y Tecnología (CONACYT) de México en el marco del proyecto de Cátedras "Infraestructura para Agilizar el Desarrollo de Sistemas Centrados en el Usuario" (Ref. 3053). Agradecemos a CONACYT por la beca de Doctorado número 743385 del primer autor, así como a la Universidad Veracruzana por el apoyo para el desarrollo de esta investigación. 


\section{REFERENCIAS}

Abdelguerfi, M., Chaudhry, N. A. \& Shaw, K., 2005. 'Stream data management'.

Adedoyin-Olowe, M., Gaber, M. M. and Stahl, F. (2013) 'A survey of data mining techniques for social media analysis', arXiv preprint arXiv:1312.4617.

Alothali, E., Alashwal, H. and Harous, S. (2019) 'Data stream mining techniques: a review', TELKOMNIKA (Telecommunication Computing Electronics and Control), 17(2), p. 728. doi: 10.12928/telkomnika.v17i2.11752.

Cheng, J., Ke, Y. and Ng, W. (2008) 'A survey on algorithms for mining frequent itemsets over data streams', Knowledge and Information Systems, 16(1), pp. 1-27. doi: 10.1007/s10115-007-0092-4.

Ellis, C. A., Gibbs, S. J. and Rein, G. L. (1991) '38 January 1991/Vol.34, No.1/COMMUNICATIONS OF THE ACM', Communications of the ACM, 34(1), pp. 39-58.

Gaber, M. M. (2005) 'Mining Data Streams: A Review', SIGMOD Rec. doi: 10.1111/j.1601-5223.1972.tb01058.x.

Gaber, M. M. et al. (2014) 'Data stream mining in ubiquitous environments: State-of-the-art and current directions', Wiley Interdisciplinary Reviews: Data Mining and Knowledge Discovery, 4(2), pp. 116-138. doi: 10.1002/widm.1115.

Gama, J. (2012) 'A survey on learning from data streams: Current and future trends', Progress in Artificial Intelligence, 1(1), pp. 45-55. doi: 10.1007/s13748-011-0002-6.

Ghesmoune, M., Lebbah, M. and Azzag, H. (2016) 'State-of-the-art on clustering data streams', Big Data Analytics. Big Data Analytics, 1(1), pp. 1-27. doi: 10.1186/s41044-016-0011-3.

Gomes, H. M. et al. (2017) 'A survey on ensemble learning for data stream classification', ACM Computing Surveys, 50(2). doi: $10.1145 / 3054925$.

Han, J. et al. (2007) 'Frequent pattern mining: Current status and future directions', Data Mining and Knowledge Discovery, 15(1), pp. 55-86. doi: 10.1007/s10618-006-0059-1.

Hulten, G., Spencer, L. and Domingos, P., 2001. 'Mining time-changing data streams', Proceedings of the seventh acm sigkdd international conference on knowledge discovery, p. 97-106.

Ikonomovska, E., Loskovska, S. and Gjorgjevik, D. (2007) 'A Survey of Stream Data Mining', 8th National Conference with International Participation, ETAI 2007, (September 2007), pp. I6-2.

Jiang, N. and Gruenwald, L., (2006). Research issues in data stream association rule mining. ACM Sigmod Record, 35(1), p. 14-19.

Kitchenham, B. A., Budgen, D. and Pearl Brereton, O. (2011) 'Using mapping studies as the basis for further research A participant-observer case study', Information and Software Technology. Elsevier B.V., 53(6), pp. 638-651. doi: 10.1016/j.infsof.2010.12.011.

Kolajo, T., Daramola, O. and Adebiyi, A. (2019) 'Big data stream analysis: a systematic literature review', Journal of Big Data. Springer International Publishing, 6(1). doi: 10.1186/s40537-019-0210-7.

Koudas, N. \& Srivastava, D., 2005. 'Data stream query processing'. Icde, Vol. 5, p. p. 1145.

Krawczyk, B. et al. (2017) 'Ensemble learning for data stream analysis: A survey’, Information Fusion, 37, pp. 132-156. doi: 10.1016/j.inffus.2017.02.004.

Liu, H., Lin, Y. and Han, J. (2011) 'Methods for mining frequent items in data streams: An overview', Knowledge and Information Systems, 26(1), pp. 1-30. doi: 10.1007/s10115-009-0267-2.

Manerikar, N. and Palpanas, T. (2009) 'Frequent items in streaming data: An experimental evaluation of the state-of-the-art', Data and Knowledge Engineering. Elsevier B.V., 68(4), pp. 415-430. doi: 10.1016/j.datak.2008.11.001.

Mohammadi, M. et al. (2018) 'Deep learning for IoT big data and streaming analytics: A survey', IEEE Communications Surveys and Tutorials. IEEE, 20(4), pp. 2923-2960. doi: 10.1109/COMST.2018.2844341.

Molero, G. and Meda, M. E. (2010) 'Integración de minería de datos y sistemas multiagente: un campo de investigación y desarrollo’. Ciencias de la Información, 41(3), p. 53-56.

Nasreen, S. et al. (2014) 'Frequent pattern mining algorithms for finding associated frequent patterns for data streams: A survey', Procedia Computer Science. Elsevier Masson SAS, 37, pp. 109-116. doi: 10.1016/j.procs.2014.08.019.

Nguyen, H. L., Woon, Y. K. and Ng, W. K. (2015) ‘A survey on data stream clustering and classification’, Knowledge and Information Systems. Springer London, 45(3), pp. 535-569. doi: 10.1007/s10115-014-0808-1.

Petersen (2017) 'Systematic Mapping Studies in Software Engineering', 58(6), pp. 484-489. doi: 10.1016/j.pedneo.2016.08.011. 